\title{
Neue Leitlinien zur Wiederbelebung
}

\section{Empfehlungen sichern die Qualität über die gesamte Rettungskette}

Die neuen Leitlinien zur kardiopulmonalen Reanimation wurden vom European Resuscitation Council (ERC) erstellt und sind auch in deutscher Sprache erhältlich. Während zentrale Aussagen zur Durchführung einer Reanimation im Wesentlichen beibehalten wurden, haben sich im Vergleich zu den Leitlinien aus dem Jahr 2010 viele Bewertungen und Details geändert. Der entscheidende Weg zu größerem Erfolg und zu mehr Überleben führt über mehr ausgebildete Ersthelfer, intelligente Alarmierungssysteme und eine deutliche Zunahme der Laienreanimation. Großer Wert wird auf die Telefonreanimation gelegt. Ein besonderes Projekt ist auch die Schülerausbildung, für die der Deutsche Rat für Wiederbelebung (GRC) ein Konzept erstellt hat.

Die Experten setzen auf "Handarbeit" „Eine manuelle Reanimation ist mindestens genauso effektiv wie die Verwendung mechanischer Reanimationshilfen“, erläutert Professor Bernd W. Böttiger, Vorsitzender des GRC und Direktor der Klinik für Anästhesiologie und Operative Intensivmedizin der Universitätsklinik Köln, Teile der neuen Leitlinie. „In einigen Studien fand sich sogar ein schlechteres neurologisches Ergebnis bei Verwendung mechanischer Systeme“, so der Experte weiter. Der Einsatz von Reanimationshilfen führe unvermeidlich zu einer gewissen Unterbrechung der Thoraxkompressionen, die so kurz wie möglich sein müssten. Die neuen Leitlinien empfehlen daher den Einsatz solcher Geräte in besonderen Situationen, wie bei Reanimationen während eines Transportes, bei sehr langer Reanimationsdauer und natürlich im Herzkatheterlabor.

Die wichtigsten Empfehlungen Zur Reanimation bei Kreislaufstillstand empfehlen die Experten eine Drucktiefe von ungefähr 5 und nicht mehr als $6 \mathrm{~cm}$. Die Frequenz soll bei 100 bis $120 / \mathrm{min}$ liegen. „Pausen von über 10 sek führen zu einer Verschlechterung der Prognose des Patienten und müssen daher vermieden werden“, erklärt Dr. Dr. Burkhard Dirks, Altvorsitzender des GRC. Adrenalin wird weiterhin empfohlen. Experten sollen eine Intubation vornehmen - wenn möglich, ohne dabei die Herzdruck- massage zu unterbrechen. Als Alternativen gelten supraglottische Atemwegshilfen. Die Kapnografie ist obligat. Innerklinisch sollten Notfallteams etabliert werden, die bei definierten Zuständen alarmiert werden und so einen Kreislaufstillstand verhindern können. Mögliche reversible Ursachen eines Kreislaufstillstandes müssen immer mit bedacht werden.

Weiterbehandlung in Spezial-Zentren Nach prähospitalem Kreislaufstillstand sind die Überlebenschancen höher, wenn die Patienten - im Einzelfall sogar unter laufender Reanimation - in spezielle Zentren (sogenannte Cardiac Arrest Center), die eine höhere Fallzahl aufweisen und eine Möglichkeit zur akuten Koronarintervention haben, eingeliefert werden.

Temperaturmanagement Nach Kreislaufstillstand bewusstlose Patienten sollen unabhängig vom initialen Herzrhythmus für mindestens $24 \mathrm{~h}$ auf $33^{\circ} \mathrm{C}$ oder $36^{\circ} \mathrm{C}$ gekühlt werden. Fieber müsse ebenso wie eine Hyperoxie in jedem Fall für $72 \mathrm{~h}$ vermieden werden. Eine Prognostizierung erscheint, so die neuen Handlungsempfehlungen, frühestens nach $72 \mathrm{~h}$ sinnvoll.

Telefonreanimation Leitstellendisponenten sollen Laien am Notruftelefon in Herzdruckmassage instruieren. „Dies ist extrem effektiv - man muss es 7-mal machen um ein Leben zusätzlich zu retten!“, erklärt Professor Karl Heinrich Scholz, stellvertretender Vorsitzender des GRC vom St. BernwardKrankenhaus in Hildesheim. In Deutschland wird die Telefonreanimation von immer mehr Leitstellen durchgeführt. In Bayern ist sie bereits landesweit verpflichtend. Auch intelligente Gesamtsysteme, in denen Ersthelfer in der Nähe per Smartphone gleichzeitig mit dem Rettungsdienst alarmiert werden, können Vorteile bringen.

Für Laien muss die Reanimation selbstverständlich sein In 50 bis $70 \%$ der Fälle beobachten Laien einen Kreislaufstillstand und sind Zeugen. Diese sollten verstärkt in Wiederbelebung ausgebildet werden, so die Empfehlungen der neuen Leitlinie. Dazu gehören die Herzdruckmassage und die Beatmung im Verhältnis 30:2. Besonderer Wert wird auf die Ausbildung von Schülern gelegt. Eine Doppelstunde pro Jahr ab der 7. Klasse sei ausreichend. Die Schüler können von speziell ausgebildeten Lehrern unterrichtet werden. Entsprechende Empfehlungen werden von der Kultusministerkonferenz 2014 und seit diesem Jahr auch von der Weltgesundheitsorganisation (WHO) unterstützt. Ein Ausbildungskonzept kommt vom GRC. „Gemeinsam 10000 Leben zusätzlich pro Jahr in Deutschland retten“, so lautet das interdisziplinär und interprofessionell formulierte Ziel, das durch die Umsetzung der Empfehlungen der neuen Leitlinien erreicht werden kann.

Nach einer Pressemitteilung des Deutschen Rat zur Wiederbelebung (GRC)

\section{Info}

Die englische Originalfassung der Leitlinie finden Sie unter www.erc.edu.

Die deutsche Übersetzung finden Sie unter www.grc-org.de/leitlinien.

Beitrag online zu finden unter http://dx.doi.org/ 10.1055/s-0035-1566274 